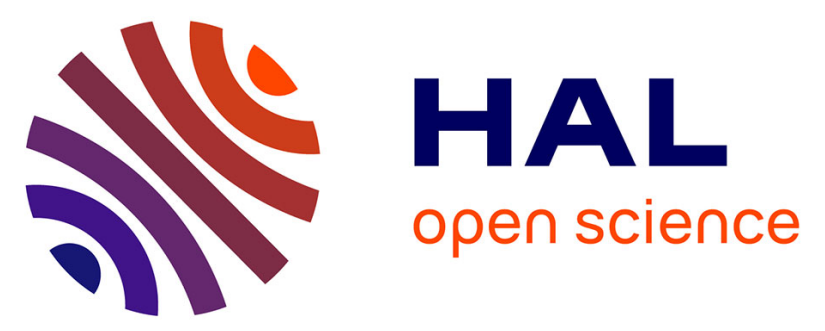

\title{
Implication of the SH3TC2 gene in Charcot-Marie-Tooth disease associated with deafness and/or scoliosis: Illustration with four new pathogenic variants
}

J. Lerat, C. Magdelaine, A. Lunati, H. Dzugan, C. Dejoie, M. Rego, P. Beze Beyrie, E. Bieth, P. Calvas, P. Cintas, et al.

\section{To cite this version:}

J. Lerat, C. Magdelaine, A. Lunati, H. Dzugan, C. Dejoie, et al.. Implication of the SH3TC2 gene in Charcot-Marie-Tooth disease associated with deafness and/or scoliosis: Illustration with four new pathogenic variants. Journal of the Neurological Sciences, 2019, 406, pp.116376. 10.1016/j.jns.2019.06.027 . hal-03271668

\section{HAL Id: hal-03271668 \\ https://hal.science/hal-03271668}

Submitted on 21 Dec 2021

HAL is a multi-disciplinary open access archive for the deposit and dissemination of scientific research documents, whether they are published or not. The documents may come from teaching and research institutions in France or abroad, or from public or private research centers.
L'archive ouverte pluridisciplinaire HAL, est destinée au dépôt et à la diffusion de documents scientifiques de niveau recherche, publiés ou non, émanant des établissements d'enseignement et de recherche français ou étrangers, des laboratoires publics ou privés.

\section{(c) (1) $\$$}

Distributed under a Creative Commons Attribution - NonCommerciall 4.0 International 


\section{Implication of the SH3TC2 gene in Charcot-Marie-Tooth disease associated with deafness and/or scoliosis: illustration with four new pathogenic}

\section{variants}

J. Lerat ${ }^{1,2}$, C. Magdelaine ${ }^{2,3}$,A. Lunati ${ }^{2,3}$, H. Dzugan ${ }^{2,3}$, C. Dejoie ${ }^{3}$, M. Rego ${ }^{3}$, E. Bieth ${ }^{4}$, P. Calvas ${ }^{4}$, P.

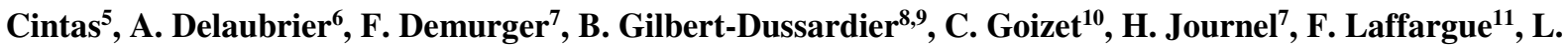

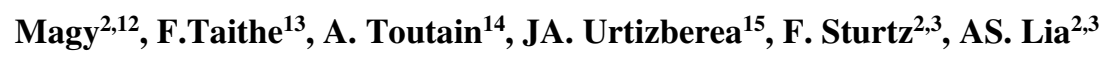

${ }^{1}$ Service Oto-rhino-laryngologie, Centre hospitalier Universitaire de Limoges, Limoges, France,

${ }^{2}$ EA6309, Université de Limoges, Limoges, France

${ }^{3}$ Service de Biochimie et Génétique Moléculaire, Centre hospitalier Universitaire de Limoges, Limoges, France

${ }^{4}$ Service de Génétique Médicale, Centre hospitalier Universitaire de Toulouse, Toulouse, France,

${ }^{5}$ Service de Neurologie et d'explorations fonctionnelles, Centre hospitalier Universitaire de Toulouse, Toulouse, France,

${ }^{6}$ Service de Médecine Physique et Rééducation, Centre hospitalier Universitaire de Poitiers, Poitiers, France,

${ }^{7}$ Service de Génétique Médicale, Centre hospitalier Bretagne Atlantique, Vannes, France

${ }^{8}$ Service de Génétique Médicale, Centre hospitalier Universitaire de Poitiers, Poitiers, France,

${ }^{9}$ EA3808, Université de Poitiers, Poitiers, France

${ }^{10}$ Service de Neurogénétique, Centre hospitalier Universitaire de Bordeaux, Bordeaux, France

${ }^{11}$ Service de Génétique médicale, Centre hospitalier Universitaire de Clermont-Ferrand, Limoges, France

${ }^{12}$ Service de Neurologie, Centre hospitalier Universitaire de Limoges, Limoges, France

${ }^{13}$ Service de Neurologie, Centre hospitalier Universitaire de Clermont-Ferrand, Limoges, France

${ }^{14}$ Service de Génétique, Centre hospitalier Universitaire de Tours, Tours, France

${ }^{15}$ Centre de Compétence Neuromusculaire, APHP, Filnemus, Centre hospitalier Hendaye, France

Corresponding author: Dr Justine LERAT 
ORL

Hôpital Mère-Enfant

8 avenue Dominique Larrey

87042 Limoges Cedex

Tel : $0033555058781 \quad$ Fax : 0033555056287

justine.lerat@chu-limoges.fr_ORCID:0000-0002-6228-7591

\section{ABSTRACT}

The autosomal recessive demyelinating form of Charcot-Marie-Tooth can be due to SH3TC2 gene pathogenic variants (CMT4C, AR-CMTde-SH3TC2). We report on a series of 13 patients with AR-CMTde-SH3TC2 among a French cohort of 350 patients suffering from all type of inheritance peripheral neuropathy. The SH3TC2 gene appeared to be the most frequently mutated gene for demyelinating neuropathy in this series by NGS. Four new pathogenic variants have been identified: two nonsense variants (p.(Tyr970*), p.(Trp1199*)) and two missense variants (p.(Leu1126Pro), p.(Ala1206Asp)). The recurrent variant p.Arg954* was present in 62\%, and seems to be a founder mutation. The phenotype is fairly homogeneous, as all these patients, except the youngest ones, presented scoliosis and/or hearing loss.

KEY WORDS: Charcot-Marie-Tooth; Neuropathy; Hearing loss; Scoliosis; SH3TC2; NGS 


\section{Introduction}

Charcot-Marie-Tooth disease (CMT) is the most frequent inherited peripheral neuropathies (1/2500). So far, variants in more than 90 genes have been identified causing either the demyelinating or the axonal form. Duplication of the PMP22 gene is the most frequent cause of the autosomal dominant demyelinating form. The autosomal recessive demyelinating form is foremost due to SH3TC2 gene pathogenic variants (CMT4C, or ARCMTde-SH3TC2) [1]. Patients with AR-CMTde-SH3TC2 suffer from early severe neuropathy starting in the first decade. Scoliosis and cranial nerve involvement, including hearing loss (HL), are frequently observed $[2,3,4]$

We report on a series of 13 patients with AR-CMTde-SH3TC2 among a French cohort of 350 patients suffering from all type of inheritance peripheral neuropathy. Phenotype-genotype correlations of these specific features have been looked for.

\section{Materials and Methods}

After giving their informed consent, 350 French patients suffering from inherited peripheral neuropathy were screened by $P M P 22$ multiplex-ligation-dependent-probe-amplification, followed by targeted next-generationsequencing using a 92-gene custom panel designed for the diagnosis of CMT and associated neuropathies (detailed in [5]; Supplementary data).

Patients were selected from diagnostic registries of a French genetic reference center. Previously, a clinical questionnaire has been fulfilled. Patients' ascertainment could be precised thanks to medical records.

The NGS panel included the 44 known CMT genes, 27 genes involved in HSN (Hereditary Sensitive Neuropathy) and HMN (Hereditary Motor Neuropathy) and 21 other genes of interest involved in neuropathies of differential diagnosis. The amplified library was prepared with Ion P1 HiQ Template OT2 200 kit (Ampliseq Custom (Life technologies)), sequenced on Proton sequencer (Life technologies), and mapped to the human reference sequence hg19/GHCh37. Variants were evaluated with Alamut Mutation Interpretation Software (Interactive Biosoftware, Rouen, France) using the NM_024577.3 reference sequence for the SH3TC2 gene. Databases such as ExAC Genome browser (http://exac.broadinstitute.org), dbSNP135 (National Center for Biotechnology Information [NCBI], Bethesda, Maryland, USA, http://www.ncbi.nlm.nih.gov/projects/SNP/), Clin Var (www.ncbi.nlm.nih.gov/clinvar), HGMD professional (www.hgmd.cf.ac.uk) and Mutalyzer 
(https://mutalyzer.nl/) were also screened. Pathogenic variants of interest were verified by Sanger sequencing using forward and reverse primer pairs.

For HL screening, MLPA and Sanger sequencing for GJB2 and GJB6 were performed.

\section{Results and discussion}

Diagnosis was positive for 201 patients $(57 \%)$. As expected, the most frequent pathogenic variant was $P M P 22$ duplication detected in 52 patients (15\%). Deletion of PMP22 was observed in 29 patients (8\%) and pathogenic point variant were detected in 120 patients (34\%).

Among these 120 patients diagnosed with point variants, 40 patients presented with a demyelinating neuropathy and the SH3TC2 gene appeared to be the most frequently mutated with 13 diagnosed patients (32.5\%). Details of their phenotypes and genotypes are presented in Tables 1 and 2.

\section{Phenotype:}

Although some clinical information is missing, we can see that all these patients, except for the youngest patients in our series, presented with scoliosis $(n=10 ; 77 \%)$ and/or deafness $(n=8 ; 62 \%)$. The phenotype is fairly homogeneous with sensori-motor demyelinating polyneuropathy with early onset before the age of 10 , except for one patient with adulthood onset (patient XII).

Sensory ataxia with poor imbalance seems to be a prominent feature, as it was found in nine patients (70\%), like Gooding et al [6].

Cranial nerve involvement is another key point of CMT4C, as it is shown in our study with eight patients reporting HL and one patient with increased latencies of visual brainstem responses. Recently, Kontogeorgiou et al reported cranial nerve involvement in 31\% of the cases [7], and Yger et al in a French cohort in 71\% [4]. HL is the foremost observed condition [8].

Pes cavus was found in eleven cases $(85 \%)$. Foot deformities are very frequently described in this phenotype $[9 ; 10]$.

Only one patient had a proximal muscle involvement, whereas it has been reported in recent studies $[11 ; 12]$. 


\begin{tabular}{|c|c|c|c|c|c|c|c|c|c|c|c|c|c|c|c|c|}
\hline \multicolumn{2}{|c|}{ Patient } & \multicolumn{8}{|c|}{ Polyneuropathy } & \multicolumn{3}{|c|}{ Hearing loss } & \multicolumn{2}{|c|}{ Scoliosis } & \multirow{2}{*}{$\begin{array}{c}\text { Cranial } \\
\text { nerve } \\
\text { involve } \\
\text { ment }\end{array}$} & \multirow{2}{*}{$\begin{array}{c}\text { Other } \\
\text { sympt } \\
\text { oms }\end{array}$} \\
\hline $\begin{array}{l}\text { Refere } \\
\text { nce } \\
\text { Famil } \\
\text { y }\end{array}$ & $\begin{array}{l}\text { Patient } \\
\text { (gender } \\
\text { /age in } \\
\text { years) }\end{array}$ & $\begin{array}{l}\text { Neuropat } \\
\text { hy }\end{array}$ & $\begin{array}{l}\text { Med } \\
\text { ian } \\
\text { nerv } \\
\text { e } \\
\text { moto } \\
\mathbf{r} \\
\text { veloc } \\
\text { ity } \\
(\mathbf{m} / \mathbf{s})\end{array}$ & $\begin{array}{l}\text { Age } \\
\text { at } \\
\text { onse } \\
\text { t } \\
\text { (yea } \\
\text { rs) }\end{array}$ & $\begin{array}{l}\text { Sensory } \\
\text { nerve } \\
\text { imapair } \\
\text { ment }\end{array}$ & $\begin{array}{l}\text { Mild } \\
\text { or } \\
\text { severe } \\
\text { weakn } \\
\text { ess }\end{array}$ & $\begin{array}{l}\text { Proxim } \\
\text { al } \\
\text { muscle } \\
\text { involve } \\
\text { ment }\end{array}$ & $\begin{array}{l}\text { Pes } \\
\text { Cav } \\
\text { us }\end{array}$ & $\begin{array}{l}\text { Loss of } \\
\text { ambula } \\
\text { tion/ } \\
\text { sensory } \\
\text { ataxia }\end{array}$ & $\begin{array}{l}\text { Pres } \\
\text { ent }\end{array}$ & $\begin{array}{l}\text { Degree } \\
\text { of } \\
\text { severity }\end{array}$ & $\begin{array}{l}\text { Age } \\
\text { at } \\
\text { ons } \\
\text { et } \\
\text { (ag } \\
\text { e in } \\
\text { yea } \\
\text { rs) }\end{array}$ & $\begin{array}{l}\text { Pres } \\
\text { ent }\end{array}$ & $\begin{array}{l}\text { Degr } \\
\text { ee of } \\
\text { sever } \\
\text { ity }\end{array}$ & & \\
\hline $\begin{array}{l}\text { Patien } \\
\text { t I }\end{array}$ & $\mathrm{F}, 22$ & $\begin{array}{l}\text { Sensori- } \\
\text { motor } \\
\text { demyelin } \\
\text { ating }\end{array}$ & 28 & 3 & $\mathbf{Y}$ & $\begin{array}{l}\text { Mode } \\
\text { rate }\end{array}$ & $\mathrm{N}$ & $\mathbf{Y}$ & $\mathrm{N} / \mathrm{Y}$ & $\mathbf{Y}$ & $\begin{array}{l}\text { Moder } \\
\text { ate }\end{array}$ & 16 & $\mathbf{Y}$ & $\mathrm{NC}$ & $\mathrm{NC}$ & $\begin{array}{l}\text { Sphin } \\
\text { cter } \\
\text { disord } \\
\text { ers }\end{array}$ \\
\hline $\begin{array}{l}\text { Patien } \\
\text { t II }\end{array}$ & M, 24 & $\begin{array}{l}\text { Sensori- } \\
\text { motor } \\
\text { demyelin } \\
\text { ating }\end{array}$ & 30 & $<8$ & $\mathbf{Y}$ & $\begin{array}{l}\text { Mode } \\
\text { rate }\end{array}$ & $\mathrm{N}$ & $\mathbf{Y}$ & $\mathrm{N} / \mathrm{N}$ & $\mathrm{N}$ & 1 & 1 & $\mathbf{Y}$ & $24^{\circ}$ & $\mathrm{N}$ & I \\
\hline $\begin{array}{l}\text { Patien } \\
\text { t III }\end{array}$ & M, 43 & $\begin{array}{l}\text { Sensori- } \\
\text { motor } \\
\text { demyelin } \\
\text { ating }\end{array}$ & 35 & 8 & $\mathbf{Y}$ & $\begin{array}{l}\text { Mode } \\
\text { rate }\end{array}$ & $\mathrm{N}$ & $\mathbf{Y}$ & $\mathrm{N} / \mathrm{Y}$ & $\mathbf{Y}$ & $\mathrm{NC}$ & $\mathrm{NC}$ & $\mathbf{Y}$ & $\begin{array}{l}\text { Seve } \\
\text { re }\end{array}$ & $\mathrm{N}$ & \\
\hline $\begin{array}{l}\text { Patien } \\
\text { t IV }\end{array}$ & $\mathrm{F}, 23$ & $\begin{array}{l}\text { Sensori- } \\
\text { motor } \\
\text { demyelin } \\
\text { ating }\end{array}$ & 25 & 1 & $\mathbf{Y}$ & $\begin{array}{l}\text { Mode } \\
\text { rate }\end{array}$ & $\mathrm{N}$ & $\mathbf{Y}$ & $\mathrm{N} / \mathrm{Y}$ & $\mathrm{N}$ & 1 & 1 & $\mathbf{Y}$ & Mild & $\mathrm{NC}$ & I \\
\hline $\begin{array}{l}\text { Patien } \\
\text { t V }\end{array}$ & $\mathrm{F}, 47$ & $\begin{array}{l}\text { Sensori- } \\
\text { motor } \\
\text { demyelin } \\
\text { ating }\end{array}$ & 25 & 4 & $\mathbf{Y}$ & $\begin{array}{l}\text { Sever } \\
\mathrm{e}\end{array}$ & $\mathrm{N}$ & $\mathbf{Y}$ & $\mathrm{N} / \mathrm{Y}$ & $\mathbf{Y}$ & Severe & $\mathrm{NC}$ & $\mathrm{N}$ & 1 & $\begin{array}{l}\text { Latenc } \\
\text { y of } \\
\text { Visual } \\
\text { brainst } \\
\text { em } \\
\text { respons } \\
\text { es } \\
\end{array}$ & I \\
\hline $\begin{array}{l}\text { Patien } \\
\text { t VI }\end{array}$ & $\mathrm{F}, 29$ & $\begin{array}{l}\text { Sensori- } \\
\text { motor } \\
\text { demyelin } \\
\text { ating }\end{array}$ & 25 & 6 & $\mathrm{NC}$ & $\begin{array}{l}\text { Sever } \\
\text { e }\end{array}$ & $\mathrm{N}$ & $\mathrm{N}$ & $\mathrm{N} / \mathrm{Y}$ & $\mathrm{NC}$ & $\mathrm{NC}$ & $\mathrm{NC}$ & $\mathbf{Y}$ & $\mathrm{NC}$ & $\mathrm{NC}$ & I \\
\hline $\begin{array}{l}\text { Patien } \\
\text { t VII }\end{array}$ & M, 56 & $\begin{array}{l}\text { Sensori- } \\
\text { motor } \\
\text { demyelin } \\
\text { ating }\end{array}$ & 32 & $\begin{array}{c}\text { Chil } \\
\text { d } \\
\text { hoo } \\
\text { d }\end{array}$ & $\mathbf{Y}$ & $\begin{array}{l}\text { Mode } \\
\text { rate }\end{array}$ & $\mathrm{N}$ & $\mathbf{Y}$ & $\mathrm{N} / \mathrm{Y}$ & $\mathbf{Y}$ & $\begin{array}{l}\text { Moder } \\
\text { ate } \\
\text { progres } \\
\text { sive }\end{array}$ & 25 & $\mathbf{Y}$ & $\mathrm{NC}$ & $\mathrm{N}$ & I \\
\hline $\begin{array}{l}\text { Patien } \\
\text { t VIII }\end{array}$ & $\mathrm{F}, 68$ & $\begin{array}{l}\text { Sensori- } \\
\text { motor } \\
\text { demyelin } \\
\text { ating }\end{array}$ & 34 & 9 & $\mathrm{NC}$ & $\begin{array}{l}\text { Sever } \\
\mathrm{e}\end{array}$ & $\mathrm{NC}$ & $\mathbf{Y}$ & $\begin{array}{l}\mathrm{NC} / \\
\mathrm{NC}\end{array}$ & $\mathbf{Y}$ & $\begin{array}{l}\text { Moder } \\
\text { ate } \\
\text { slope } \\
\text { curve } \\
\end{array}$ & $\mathrm{NC}$ & $\mathbf{Y}$ & $\mathrm{NC}$ & $\mathrm{NC}$ & 1 \\
\hline $\begin{array}{l}\text { Patien } \\
\text { t IX }\end{array}$ & $\mathrm{F}, 12$ & $\begin{array}{l}\text { Sensori- } \\
\text { motor } \\
\text { demyelin } \\
\text { ating }\end{array}$ & 34 & 3 & $\mathbf{Y}$ & $\begin{array}{l}\text { Sever } \\
\mathrm{e}\end{array}$ & $\mathbf{Y}$ & $\mathbf{Y}$ & $\mathbf{Y} / \mathbf{Y}$ & $\mathbf{Y}$ & Mild & 11 & $\mathrm{~N}$ & 1 & $\mathrm{~N}$ & I \\
\hline $\begin{array}{l}\text { Patien } \\
\text { t X }\end{array}$ & $\mathrm{M}, 9$ & $\begin{array}{l}\text { Sensori- } \\
\text { motor } \\
\text { demyelin } \\
\text { ating }\end{array}$ & 30 & $<5$ & $\mathrm{~N}$ & $\begin{array}{l}\text { Mode } \\
\text { rate }\end{array}$ & $\mathrm{N}$ & $\mathbf{Y}$ & $\mathbf{Y} / \mathbf{Y}$ & $\mathrm{N}$ & I & 1 & $\mathbf{Y}$ & $\begin{array}{c}\text { Mild } \\
<10 \\
\circ\end{array}$ & $\mathrm{N}$ & $\begin{array}{l}\text { Autis } \\
\mathrm{m}\end{array}$ \\
\hline $\begin{array}{l}\text { Patien } \\
\text { t XI }\end{array}$ & $\mathrm{F}, 71$ & $\begin{array}{l}\text { Sensori- } \\
\text { motor } \\
\text { demyelin } \\
\text { ating }\end{array}$ & 30 & $\begin{array}{c}\text { Tee } \\
\text { ns }\end{array}$ & $\mathbf{Y}$ & $\begin{array}{l}\text { Sever } \\
\mathrm{e}\end{array}$ & $\mathrm{N}$ & $\mathbf{Y}$ & $\mathrm{N} / \mathrm{Y}$ & $\mathbf{Y}$ & $\begin{array}{c}\text { Severe } \\
\text { progres } \\
\text { sive }\end{array}$ & $\begin{array}{c}< \\
10\end{array}$ & $\mathbf{Y}$ & $\mathrm{NC}$ & $\mathrm{N}$ & $\begin{array}{l}\text { Bilate } \\
\text { ral } \\
\text { catara } \\
\text { ct }\end{array}$ \\
\hline $\begin{array}{l}\text { Patien } \\
\text { t XII }\end{array}$ & M, 83 & $\begin{array}{l}\text { Sensori- } \\
\text { motor } \\
\text { demyelin } \\
\text { ating }\end{array}$ & 31 & 73 & $\mathbf{Y}$ & $\begin{array}{l}\text { Sever } \\
\mathrm{e}\end{array}$ & $\mathrm{N}$ & $\mathrm{N}$ & $\begin{array}{l}\mathrm{NC} / \\
\mathrm{NC}\end{array}$ & $\mathbf{Y}$ & $\begin{array}{c}\text { Moder } \\
\text { ate } \\
\text { U- } \\
\text { shaped } \\
\text { curve }\end{array}$ & $\mathrm{NC}$ & $\mathbf{Y}$ & $\mathrm{NC}$ & $\mathrm{NC}$ & I \\
\hline $\begin{array}{l}\text { Patien } \\
t \text { XIII }\end{array}$ & $\mathrm{M}, 27$ & $\begin{array}{l}\text { Sensori- } \\
\text { motor } \\
\text { demyelin } \\
\text { ating }\end{array}$ & 30 & 8 & $\mathbf{Y}$ & $\begin{array}{l}\text { Mode } \\
\text { rate }\end{array}$ & $\mathrm{N}$ & $\mathbf{Y}$ & $\mathrm{N} / \mathrm{N}$ & $\mathrm{N}$ & 1 & 1 & $\mathrm{~N}$ & 1 & $\mathrm{~N}$ & $\begin{array}{l}\text { Musc } \\
\text { ular } \\
\text { pain }\end{array}$ \\
\hline
\end{tabular}


Table 1: Phenotypes of our 13 patients presenting with a demyelinating hereditary neuropathy among a cohort of 350 French patients

(F: Female; M: Male; NC: Not Communicated; /: not applicable; Y: Yes; N: No)

\section{Genotype:}

In addition to already known pathogenic variants, four new pathogenic variants have been identified: two nonsense variants (c.2910C>A, p.(Tyr970*) and c.3596G>A, p.(Trp1199*)) and two missense variants (c.3377T>C, p.(Leu1126Pro) and c.3617C>A, p.(Ala1206Asp)).

Analysis at the DFNB1 locus did not reveal any pathogenic variant for all diagnosed and known deaf patients, which is in favour of the implication of the SH3TC2 gene in hearing loss.

The recurrent variant p.Arg954* was present in $62 \%$ of our patients. This is similar to another French study by Yger et al (62.5\%) and a Czech study by Lassuthova et al with $63.2 \%$ [4; 10].

Thus, this pathogenic variant is prevalent in 5\% in Northern Europe, such as Norway [13], and is also frequent in Italy [8;14]. As it is also identified in North America, p.Arg954* seems to be a founder mutation [15;16]. Haplotype analysis should be performed to confirm this hypothesis.

\begin{tabular}{|c|c|c|c|c|c|c|c|}
\hline \multicolumn{3}{|c|}{ Patient } & \multicolumn{5}{|c|}{ Genotype } \\
\hline $\begin{array}{l}\text { Reference } \\
\text { Family }\end{array}$ & $\begin{array}{l}\text { Patient } \\
\text { (gender/age } \\
\text { in years) }\end{array}$ & Country & Mutation type & Zygosity & $\begin{array}{l}\text { Nucleotide } \\
\text { change }\end{array}$ & $\begin{array}{l}\text { Amino acid } \\
\text { change }\end{array}$ & Localization \\
\hline Patient I & $\mathrm{F}, 22$ & France & Nonsense & Homozygous & c. $2860 \mathrm{C}>\mathrm{T}$ & p.Arg954* & Exon11 \\
\hline Patient II & F, 29 & France & Nonsense & Homozygous & c. $2860 \mathrm{C}>\mathrm{T}$ & p.Arg954* & Exon11 \\
\hline Patient III & M, 43 & France & Nonsense & Homozygous & c. $2860 \mathrm{C}>\mathrm{T}$ & p.Arg954* & Exon11 \\
\hline Patient IV & $\mathrm{F}, 23$ & France & $\begin{array}{l}\text { Nonsense }+ \\
\text { Nonsense }\end{array}$ & $\begin{array}{l}\text { Compound } \\
\text { heterozygous }\end{array}$ & $\begin{array}{l}\text { c. } 2860 \mathrm{C}>\mathrm{T}+ \\
\text { c. } 3325 \mathrm{C}>\mathrm{T}\end{array}$ & $\begin{array}{l}\text { p.Arg954*+p. } \\
\text { Arg1109* }\end{array}$ & $\begin{array}{l}\text { Exon11+ } \\
\text { Exon14 }\end{array}$ \\
\hline Patient V & $\mathrm{F}, 47$ & France & Nonsense & Homozygous & c. $3325 \mathrm{C}>\mathrm{T}$ & p.Arg1109* & Exon14 \\
\hline Patient VI & F, 29 & France & $\begin{array}{l}\text { Nonsense }+ \\
\text { Nonsense }\end{array}$ & $\begin{array}{l}\text { Compound } \\
\text { heterozygous }\end{array}$ & $\begin{array}{l}\text { c. } 2860 C>T+ \\
\text { c. } 2910 C>A\end{array}$ & $\begin{array}{l}\text { p.Arg954* }+ \\
\text { p.Tyr970* }\end{array}$ & $\begin{array}{l}\text { Exon11+ } \\
\text { Exon12 }\end{array}$ \\
\hline Patient VII & M, 56 & France & Nonsense & Homozygous & c. $3321 \mathrm{C}>\mathrm{A}$ & p.Tyr1107* & Exon14 \\
\hline Patient VIII & $\mathrm{F}, 68$ & France & $\begin{array}{l}\text { Nonsense } \\
\text { Missense }\end{array}$ & $\begin{array}{l}\text { Compound } \\
\text { heterozygous }\end{array}$ & $\begin{array}{l}\text { c. } 2860 \mathrm{C}>\mathrm{T}+ \\
\text { c. } 3377 \mathrm{~T}>\mathrm{C}\end{array}$ & $\begin{array}{l}\text { p.Arg954* + } \\
\text { p.Leu1126Pro }\end{array}$ & $\begin{array}{l}\text { Exon } 11+ \\
\text { Exon } 15\end{array}$ \\
\hline Patient IX & $\mathrm{F}, 12$ & France & $\begin{array}{l}\text { Nonsense }+ \\
\text { Missense }\end{array}$ & $\begin{array}{l}\text { Compound } \\
\text { heterozygous }\end{array}$ & $\begin{array}{l}\text { c. } 2860 \mathrm{C}>\mathrm{T} \\
+\mathrm{c} .3511 \mathrm{C}>\mathrm{T}\end{array}$ & $\begin{array}{l}\text { p.Arg954* + } \\
\text { p.Arg1171Cys }\end{array}$ & $\begin{array}{l}\text { Exon11+ } \\
\text { Exon } 16\end{array}$ \\
\hline Patient X & $\mathrm{M}, 9$ & France & $\begin{array}{ll}\text { Nonsense } & + \\
\text { Missense } & \end{array}$ & $\begin{array}{l}\text { Compound } \\
\text { heterozygous }\end{array}$ & $\begin{array}{l}\text { c. } 2860 C>T+ \\
\text { c. } 3511 C>T\end{array}$ & $\begin{array}{l}\text { p.Arg954* + } \\
\text { p.Arg1171Cys }\end{array}$ & $\begin{array}{l}\text { Exon } 11+ \\
\text { Exon } 16\end{array}$ \\
\hline Patient XI & $\mathrm{F}, 71$ & France & $\begin{array}{l}\text { Missense } \quad+ \\
\text { Nonsense }\end{array}$ & $\begin{array}{l}\text { Compound } \\
\text { heterozygous }\end{array}$ & $\begin{array}{l}\text { c. } 2642 A>G+ \\
\text { c. } 3596 G>A\end{array}$ & $\begin{array}{l}\text { p.Asn881Ser + } \\
\text { p.Trp1199* }\end{array}$ & $\begin{array}{l}\text { Exon11+ } \\
\text { Exon } 16\end{array}$ \\
\hline Patient XII & $\mathrm{M}, 83$ & France & Missense & Homozygous & c. $3617 \mathrm{C}>\mathrm{A}$ & p.Ala1206Asp & Exon16 \\
\hline Patient XIII & M, 27 & France & Missense & Compound & c. $1969 \mathrm{G}>\mathrm{A}+$ & p.Glu657Lys + & Exon11+ \\
\hline
\end{tabular}


\begin{tabular}{|l|l|l|l|l|l|l|}
\hline & & Missense & heterozygous & c.2642A $>\mathrm{G}$ & p.Asn881Ser & Exon11 \\
\hline
\end{tabular} among a cohort of 350 French patients. Strong grey color corresponds to nonsense variants, middle grey color to nonsense variant associated to missense variant, mild grey color to missense variants.(F: Female; M: Male; in

Red:

novel

variants

found) 


\section{Conclusion}

SH3TC2 appears to be the most frequently implicated gene in autosomal recessive demyelinating form of CMT in the French population, often associated with scoliosis and/or HL. Sensory ataxia and pes cavus are prominent features of CMT4C. Moreover, p.Arg954* appears to be a founder mutation.

\section{Conflict of interest: None.}

Ethical approval: All procedures performed in studies involving human participants were in accordance with the ethical standards of the institutional and national research committee and with the 1964 Helsinki declaration and its later amendments or comparable ethical standards.

Acknowledgements: We are thankful to the nucleic acid platform BISCEM, University of Limoges, France. We also thank Sylvie Gautier for English revision. 


\section{References}

[1] Mathis S, Goizet C, Tazir M, Magdelaine C, Lia A-S, Magy L, Vallat JM (2015) Charcot-MarieTooth diseases: an update and some new proposals for the classification. J Med Genet 52(10):681-90.

[2] Senderek J, Bergmann C, Stendel C, Kirfel J, Verpoorten N, De Jonghe P, Timmerman V, Chrast R, Verheijen MH, Lemke G, Battaloglu E, Parman Y, Erdem S, Tan E, Topaloglu H, Hahn A, Müller-Felber W, Rizzuto N, Fabrizi GM, Stuhrmann M, Rudnik-Schöneborn S, Züchner S, Michael Schröder J, Buchheim E, Straub V, Klepper J, Huehne K, Rautenstrauss B, Büttner R, Nelis E, Zerres K (2003) Mutations in a gene encoding a novel SH3/TPR domain protein cause autosomal recessive Charcot-Marie-Tooth type 4C neuropathy. Am J Hum Genet 73(5):1106-19

[3] Azzedine H, LeGuern E, Salih MA (2008). Charcot-Marie-Tooth Neuropathy Type 4C. In: GeneReviews® (ed) Adam MP, Ardinger HH, Pagon RA, Wallace SE, Bean LJ, Stephens K, et al. 1993-2018. http://www.ncbi.nlm.nih.gov/books/NBK1340/

[4] Yger M, Stojkovic T, Tardieu S, Maisonobe T, Brice A, Echaniz-Laguna A, Alembik Y, Girard S, Cazeneuve C, Leguern E, Dubourg O (2012) Characteristics of clinical and electrophysiological pattern of Charcot-Marie-Tooth 4C. J Peripher Nerv Syst 17(1):112-22.

[5] Lerat J, Cintas P, Beauvais-Dzugan H, Magdelaine C, Sturtz F, Lia AS (2017) A complex homozygous mutation in ABHD12 responsible for PHARC syndrome discovered with NGS and review of the literature. J Peripher Nerv Syst 22(2):77-84.

[6] Gooding R, Colomer J, King R, Angelicheva D, Marns L, Parman Y, Chandler D, Bertranpetit J, Kalaydjieva L (2005) A novel Gypsy founder mutation, p.Arg1109X in the CMT4C gene, causes variable peripheral neuropathy phenotypes. J Med Genet 42(12):e69.

[7] Kontogeorgiou Z, Nikolaou K, Kartanou C, Breza M, Panas M, Karadima G, Koutsis G (2019) Mutational screening of the SH3TC2 gene in Greek patients with suspected demyelinating recessive Charcot-Marie-Tooth disease reveals a varied and unusual phenotypic spectrum. J Peripher Nerv Syst 24(1):125-130. doi: 10.1111/jns.12305. Epub 2019 Feb 6.

[8] Piscosquito G, Saveri P, Magri S, Ciano C, Gandioli C, Morbin M, Bella DD, Moroni I, Taroni F, Pareyson D (2016) Screening for SH3TC2 gene mutations in a series of demyelinating recessive Charcot-Marie-Tooth disease (CMT4). J Peripher Nerv Syst 2016 Sep;21(3):142-9. doi: 10.1111/jns.12175. 
[9] Baets J, Deconinck T, De Vriendt E, Zimoń M, Yperzeele L, Van Hoorenbeeck K, Peeters K, Spiegel R, Parman Y, Ceulemans B, Van Bogaert P, Pou-Serradell A, Bernert G, Dinopoulos A, Auer-Grumbach M, Sallinen SL, Fabrizi GM, Pauly F, Van den Bergh P, Bilir B, Battaloglu E, Madrid RE, Kabzińska D, Kochanski A, Topaloglu H, Miller G, Jordanova A, Timmerman V, De Jonghe P (2011) Genetic spectrum of hereditary neuropathies with onset in the first year of life. Brain 134(Pt 9):2664-76. doi: 10.1093/brain/awr184. Epub 2011 Aug 11.

[10] Laššuthová P, Mazanec R, Vondráček P, Sišková D, Haberlová J, Sabová J, Seeman P (2011) High frequency of SH3TC2 mutations in Czech HMSN I patients. Clin Genet 80(4):334-45. doi: 10.1111/j.13990004.2011.01640.x. Epub 2011 Mar 1.

[11] Arntzen KA, Høyer H, Ørstavik K, Tallaksen C, Vedeler C, Østern R, Nebuchennykh M, Braathen GJ, Fagerheim T (2018) Charcot-Marie-Tooth disease type 4C in Norway: Clinical characteristics, mutation spectrum and minimum prevalence. Neuromuscul Disord 28(8):639-645. doi: 10.1016/j.nmd.2018.06.004. Epub 2018 Jun 15.

[12] de Oliveira CM, Fussiger H, Winckler PB, Saute JAM (2019) Dropped head syndrome as a manifestation of Charcot-Marie-Tooth disease type 4C. Neuromuscul Disord 29(2):138-141. doi: 10.1016/j.nmd.2018.11.010. Epub 2018 Nov 29.

[13] Høyer H, Braathen GJ, Busk ØL, Holla ØL, Svendsen M, Hilmarsen HT, Strand L, Skjelbred CF, Russell MB (2014) Genetic diagnosis of Charcot-Marie-Tooth disease in a population by next-generation sequencing.Biomed Res Int 2014:210401. doi: 10.1155/2014/210401. Epub 2014 Jun 16.

[14] Manganelli F, Tozza S, Pisciotta C, Bellone E, Iodice R, Nolano M, Geroldi A, Capponi S, Mandich P, Santoro L (2014) Charcot-Marie-Tooth disease: frequency of genetic subtypes in a Southern Italy population. J Peripher Nerv Syst. 19(4):292-8. doi: 10.1111/jns.12092.

[15] Lupski JR, Gonzaga-Jauregui C, Yang Y, Bainbridge MN, Jhangiani S, Buhay CJ, Kovar CL, Wang M, Hawes AC, Reid JG, Eng C, Muzny DM, Gibbs RA (2013) Exome sequencing resolves apparent incidental findings and reveals further complexity of SH3TC2variant alleles causing Charcot-Marie-Tooth neuropathy. Genome Med. 5(6):57. doi: 10.1186/gm461. eCollection 2013.

[16] Varley TL, Bourque PR, Baker SK (2015) Phenotypic variability of CMT4C in a French-Canadian kindred. Muscle Nerve. 52(3):444-9. doi: 10.1002/mus.24640. Epub 2015 May 14. 


\section{Supplementary data}

Supp. Table S1: 92-gene panel used for NGS. It includes the 44 known CMT genes, 27 genes involved in HSN

(Hereditary Sensitive Neuropathy) and HMN (Hereditary Motor Neuropathy) and 21 other genes of interest involved in neuropathies of differential diagnoses. 